\title{
NUMERICAL STUDY ON SECONDARY JET INJECTION OF FLUID FOR THRUST VECTORING IN 2-D SUPERSONIC NOZZLE
}

\section{BINDU MOUNICA \& MURALI KRISHNA}

Research Scholar, Jawaharlal Nehru Technological University Hyderabad, India

Dean, Malla Reddy Institute of Engineering \& Technology, Hyderabad, India

\begin{abstract}
Maneuvering of high speed aircraft is a challenging part during designing of the jet aircrafts in which various proposals had come with many solutions which includes the Secondary fluidic thrust vectoring. Here the study is performed to observe the flow variation by injecting the secondary fluid at eh exit of the nozzle. In supersonic nozzles due to injection of secondary liquid we observed shock and formation of these shocks are studied well. Fluidic thrust vectoring with the shock vector control method is widely studied at the moment, requires forced, asymmetric fluidic injection of a secondary air stream into the supersonic, primary flow that develops in the divergent section of the nozzle at certain conditions. This technology also can improve aircraft maneuverability by manipulating the nozzle flow to deflect from its axial direction our main objective is to investigate the effect of a secondary jet on the primary flow in a converging-diverging nozzle, and to find the location of the FTV nozzle and angle of injection for the secondary fluid.

An oblique Shock wave generated due to interaction of secondary fluid with primary fluid is studied. This shock wave produce back pressure which can be regulated by Nozzle pressure ratio (NPR) and Secondary nozzle pressure ratio (SPR). Computations are performed with and without the secondary jet injection for different combinations of NPR, SPR, secondary jet location, and secondary angular injection. The thrust pitching moment and the thrust pitching angle are determined to evaluate the FTV performance.
\end{abstract}

KEYWORDS: Supersonic Nozzle, Secondary Injection, Thrust Vectoring, Secondary Nozzle Pressure Ratio (SPR).CFDFluen

Received: Jun 08, 2020; Accepted: Jun 28, 2020; Published: Aug 20, 2020; Paper Id.: IJMPERDJUN2020832

\section{INTRODUCTION}

With the development of high performance aero-engine, the fluidic fixed geometric nozzle gradually attracts more attention of researchers, due to its low weight, simple structure and no actuating system [1-3] and various types of FTV concepts were proposed including co-flow vector nozzle, Counter- flow vector nozzle, throat skewing vector nozzle, dual throat vector nozzle, and shock vector controlling nozzle [4-8].

The common feature for these FTV nozzles is that secondary flow is used to control the deflection of primary flow. At present a lot of studies have been conducted on the influence of geometric and aerodynamic parameters on SVC nozzle vector performance by computational and experimental investigation. The previous studies only focus on the parameters influence on the nozzle. In fact, the secondary flow for SVC nozzle to get the thrust vector is extracted from the high pressure components such as fan or HPC. The pressure and mass flow of secondary flow are all constraint on the engine state. So the ignoring of the couple between SVC nozzle and engine will lead to unreasonable result. 
In this paper, a component-based aero-engine simulation system is established based on object- oriented method for overcoming the limitation of current engine model. The characteristic of SVC nozzle with considering of its aerodynamic and geometric parameters is obtained. By employing RSM model, the couple model for the engine with SVC nozzle is formed and its performance is estimated and discussed in detail. The characteristic of certain SVC nozzle is obtained by computational fluid dynamics method. To evaluate the performance of the engine with SVC nozzle, the conceptual design of double bypass variable cycle engine with SVC nozzle is given. The influence of injecting secondary fluid in CDFS outlet and the first stage of HPC is discussed. Finally performances such as vector angle, thrust coefficient, thrust and specific fuel consumption are obtained by employing the engine simulation model built by this paper.

\section{BOUNDARY CONDITIONS}

- A static wall condition is considered for the nozzle with no slip condition.

- $\quad$ On solid surface: no-slip adiabatic boundary is applied specifying pressure as input.

- A medium of free stream is employed in between Inlet and outlet by holding particular altitude.

- $\quad$ Pressure inlet is chosen with an inlet Mach number of $\mathrm{M}=1.5$

- For secondary injection with a precise calculation's the fluid is injected with various velocities to observe the angle of vectoring

\section{COMPUTATION METHOD}

Ansys CFD is used to conduct the experiment of internal nozzle performance and fluidic thrust vectoring using Convergent divergent nozzle. Multi block feature is used to develop the grid around the nozzle. Using solvent solver in 2-D unsteady flow, Reynolds averaged Naver stokes equations, URANS equations were solved together with spalart-allmaras one equation turbulence model. Based on the previous studies Spalartallmaras and k-Ivarepsolon turbulence models have shown to be accurate popular for fluidic thrust vectoring simulations. The spalartallmaras one equation model provides the mist stable results which are favorable comparable to the experimental test. Some other researchers have chosen k-e turbulence model requiring a wall function or a damping function. The one-equation Spalartallmaras turbulence is used here.

The nozzle used in this study was symmetric, rectangular, and convergent-divergent nozzle from NASA Langley Research Center (Waitheet al., 2003). The length of the nozzle was $115.57 \mathrm{~mm}$, while the throat area of the nozzle was $2785.19 \mathrm{~mm}^{2}$. In addition, height of the throat was $27.48 \mathrm{~mm}$, and from throat to inlet was $57.78 \mathrm{~mm}$. The base area ratio of the nozzle outlet to the throat (expansion ratio) was 1.796. The nozzle inlet centre was set to be the origin of coordinates, the secondary inlet $\mathrm{w}$ a s located at $46.35 \mathrm{~mm}$ from injection slot to throat, while the width of slot was $2.032 \mathrm{~mm}$ (Fig. 3). A comparison between three different quality meshes predicted static pressure distribution.

The grid study is conducted to study the variation of output. Here we performed three grid sizes of 1,545,405 (coarse) 3,090,810 (medium) and 4,636,215 (fine), respectively. The comparisons of the obtained results demonstrate that medium and fine meshes are very close and better than coarse mesh. Since the maximum difference in the static pressure distribution (between the medium and fine meshes) is about $1.0 \%$ and the location of the main separation line is almost located at the same position for two different meshes. Thus, the computations were performed on the medium mesh to cut down the computational time. 


\section{MODELLING AND SIMULATIONS}

Complete simulation helps in proper location and size of air intake as well as combustor geometry. However a crucial factor which needs deep consideration is the nozzle flow. On other hand during supercritical condition, shock train moves in the di $\square$ user to recover pressure that may again lead to poor results in lower e $\square$ ciency. The study of cold flows gives the performance of the engine in all the sections before fuel mixture.

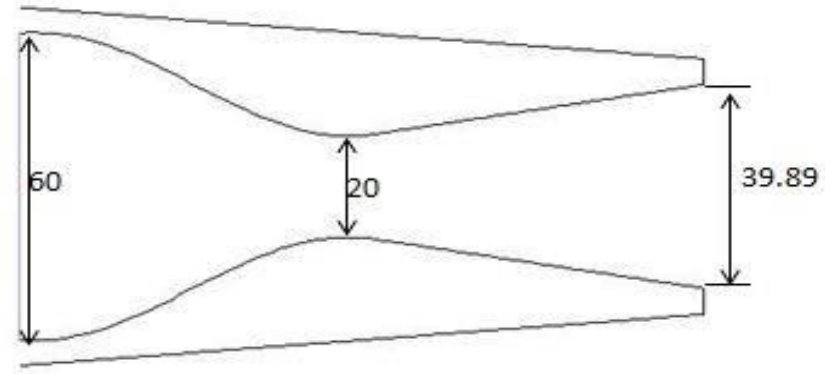

Figure 1: Preferred Geometry.
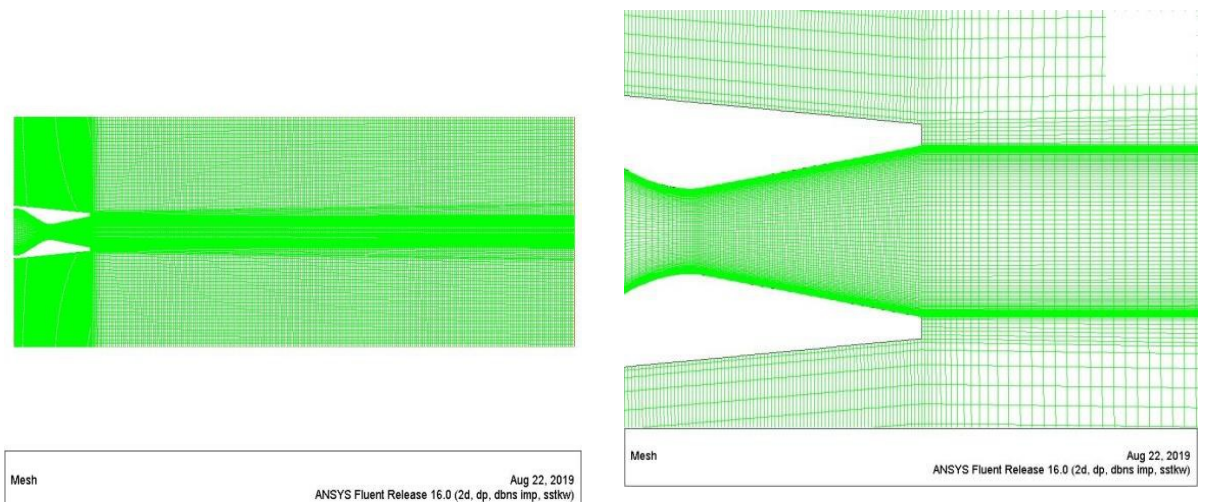

ANSYS Fluent Release 16.0 (2d, dp. Abns min, ssihini)

Figure 2: Geometry Mesh.

\section{RESULTS AND PLOTS}

\subsection{Effect of Nozzle Expansion Ratio with Secondary Injection Angle}

To understand the effects of fluidic injection angle with variable nozzle expansion ratio in vectored nozzle performance, simulations were achieved at injection at an angle of $90^{0}$, with nozzle pressure ratios of NPR=3.0 and 4.6, with different SPR (1.3). In addition, nozzle design were performed at variable expansion ratio (Ae/At =1.796) and with nozzle divergent angles of $\theta=11.01^{0}$.

The effects of fluidic injection in thrust vectoring performance improve with optimizing injection angle, decreasing NPR, and increasing SPR. The thrust vectoring angle and thrust vectoring efficiency at (Ae/At=1.796), with different NPR and SPR, as compared to optimal and normal to boundary fluidic injection angle have average increases of about $29.44 \%$ and $38.21 \%$, in turn. Comparing all configurations at NPR=4.6, the nozzle with (Ae/At=1.796) performs significantly better in terms of thrust vectoring. The current improvement can be claimed to be the result of the increase of pressure differential along nozzle surfaces.

Finally, increasing expansion ratio of nozzle with decreasing NPR, which is representative of most cases studied, has positive impact on pitch thrust vector angle and thrust vectoring efficiency. Also, increasing expansion ratio with 
increasing SPR, except for Ae/At =1.398 (achieved by normal to boundary injection angle), has positive effect on thrust vectoring performance. Moreover, increasing expansion ratio of nozzle with optimizing injection angle, which is representative of all cases studied, has positive impact on both pitch thrust vector angle and thrust vectoring efficiency.

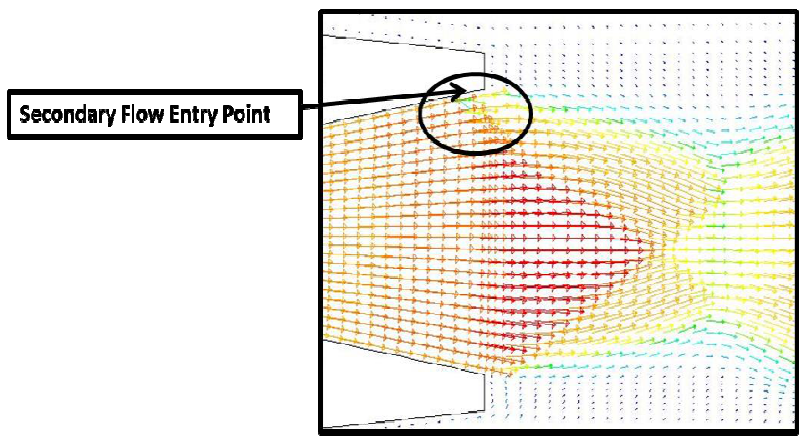

Figure 3: Secondary Injection position.
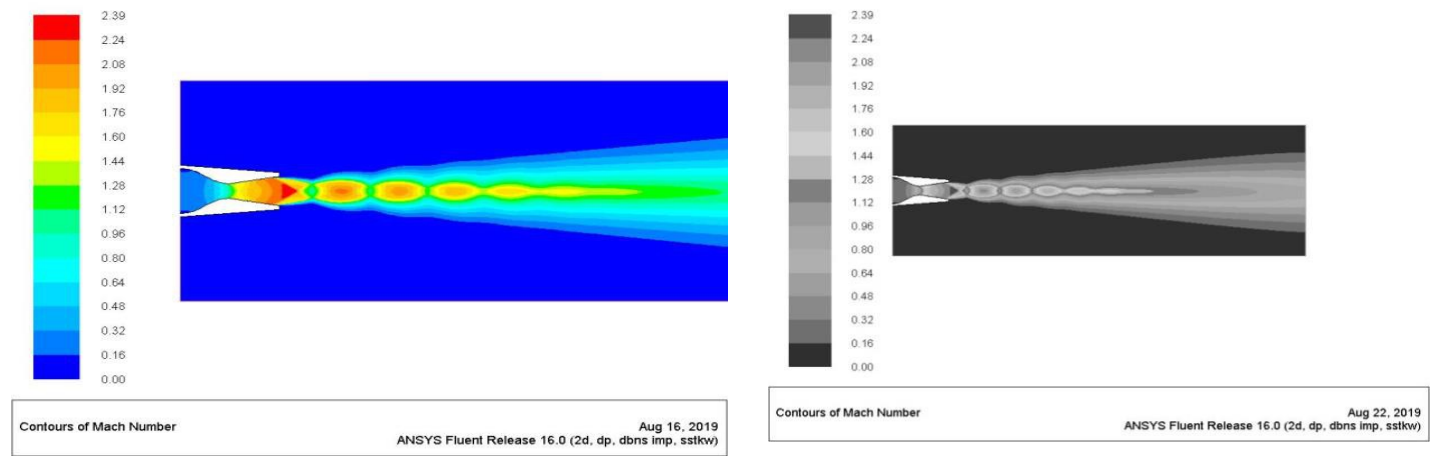

Figure 4: Mach Number Plots Before Injection.
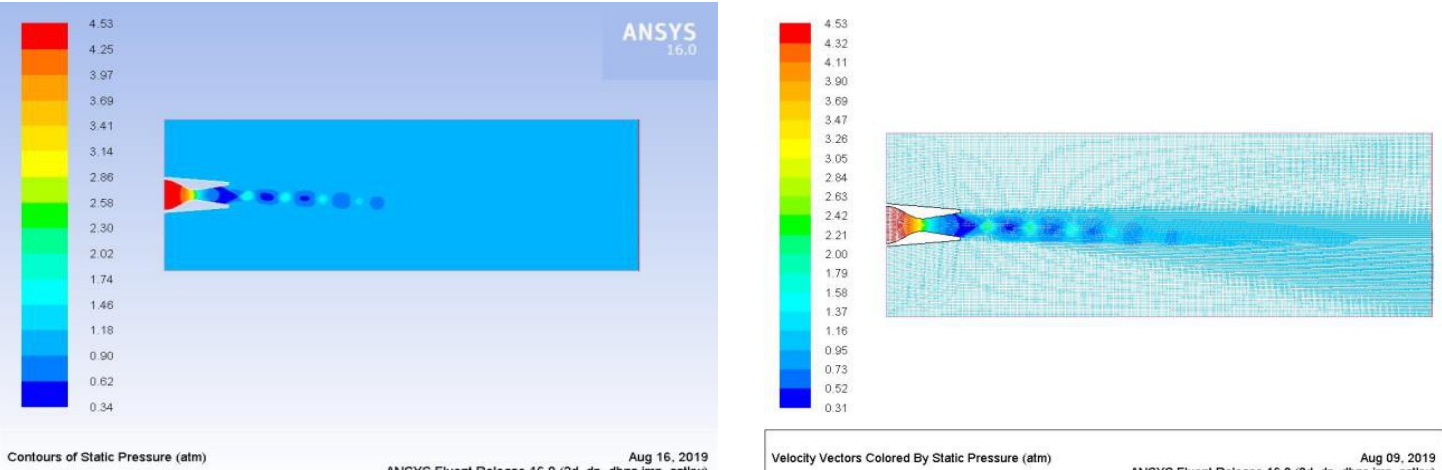

Figure 5: Mach Number Plot Before and After Vectoring.
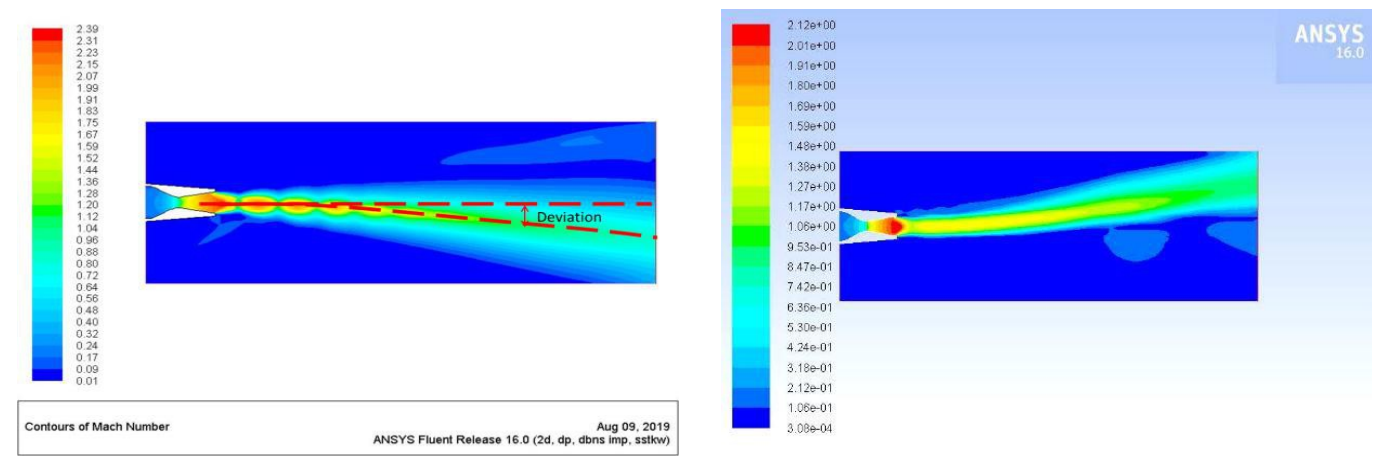

Figure 6 Magnitude of the Flow Where $\theta=$ ? 0 . 


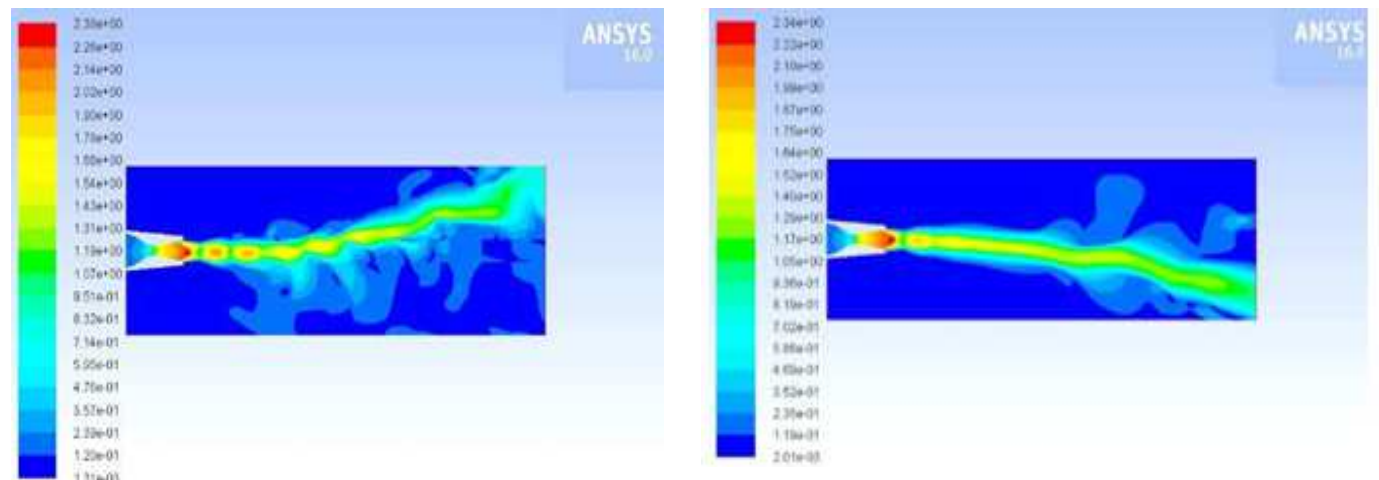

Figure 7 Thrust Vectoring When Sfc is Injection From Top Injector Thrust Vectoring When Fluid Is Injection From Tope Injector.

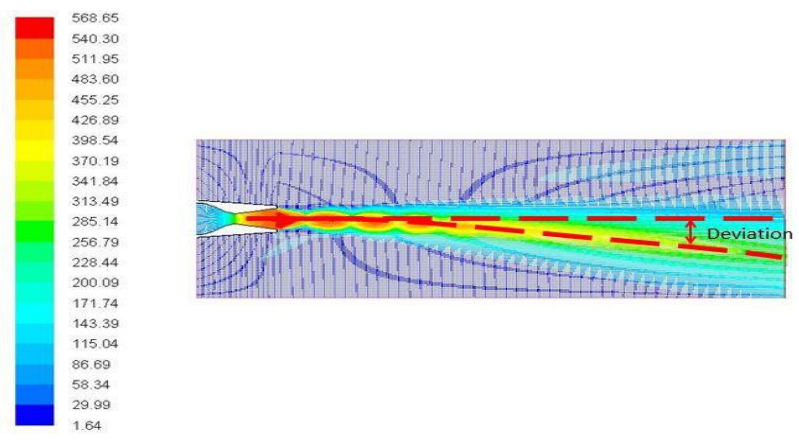

Figure 8: Angle of Vectoring by Injecting Secondary Fluid.

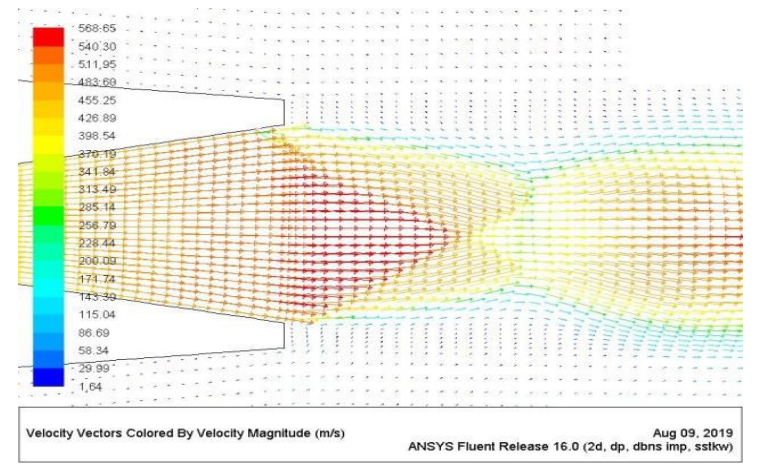

Figure 8 Direction of the Mixed Flow.

\section{CONCLUSIONS}

A computational investigation of fluidic injection angle effects on thrust vectoring (FTV) has been conducted in the current study. The results can be of invaluable assistance in FTV optimization design and operation. The influence of effective parameters on fluidic thrust vectoring including nozzle pressure ratio, secondary pressure ratio, and fluidic injection angle in normal direction is thoroughly investigated. Furthermore, nozzle design included different expansion ratios (Ae/At $=1.796)$.

\section{The Findings of the Study Have Indicated That:}

- The secondary injection angle is an essential parameter in fluidic thrust vectoring

- in all cases, optimizing secondary injection angle would have positive impact on pitch thrust vector angle and thrust vectoring efficiency (by average increases of more than $44.76 \%$ and $53.14 \%$, respectively) 3 . In most cases, 
increasing NPR has negative impact on both pitch thrust vector angle and thrust vectoring efficiency over the range of the studied NPR.

- The effect of decreasing total pressure of nozzle (by decreasing NPR), would increase pitch thrust vector angle and thrust vectoring efficiency.

- In most cases with constant NPR, increasing SPR would increase pitch thrust vector angle and decrease thrust vectoring efficiency by decreasing the effective area in the nozzle

- In most cases, increasing expansion ratio of the nozzle with decreasing NPR has positive impact on pitch thrust vector angle and thrust vectoring efficiency.

\section{REFERENCES}

1. Karen A. Deere, Summary of fluidic thrust vectoring research conducted at NASA Langley research centre, $21^{\text {st }}$ applied aerodynamics conference, 23-26 June 2003, Oriando, Florida. AIAA 20033800.

2. M. S. Mason, W. J. Crowther, Fluidic thrust vectoring for low observable air vehicle, 2nd AIAA flow control conference, 28 June-1 July 2004, Portiand. Oregon, AIAA 20042210.

3. Yvette S. Weber, Douglas L. Bowers, Advancements in exhaust system technology for the $21^{\text {st }}$ century, AIAA-983100.

4. Khan, Sher Afghan, Abdul Aabid, and Maughal Ahmed Ali Baig. "CFD analysis of CD nozzle and effect of nozzle pressure ratio on pressure and velocity for suddenly expanded flows." International Journal of Mechanical and Production Engineering Research and Development 8 (2018): 11471158.

5. AfshinBanazadeh, FariborzSaghafi, Multi-directional Co-flow fluidic thrust vectoring intended for a small gas turbine, AIAA 2007 conference and exhibt, 7-10 May 2007, Rohnert Park, California. AIAA 20072940.

6. Strykowshi, P. J., Krothapalli, A., The counter current mixing layer: strategies for shear-layer control, AIAA shear flow conference, July 6-9,1993, Orlando, FL. AIAA 933260.

7. BHAKTA, SHUBHANKAR, and BHUPINDER SINGH."NUMERICAL STUDY OF CAVITY-BASED FLAME-HOLDER WITH SLOT INJECTION FOR SUPERSONIC COMBUSTION."International Journal of Mechanical and Production Engineering Research and Development (IJMPERD) 8. 2, Apr 2018, 887898.

8. D. N. Miller, P. J. Yagle. J. W. Hamstra, Fluidic throat skewing for thrust vectoring in fixed geometry nozzles, $37^{\text {th }}$ AIAA aerospace sciences meeting and exhibit, January 11,19991 Reno, NV. AIAA9916262.

9. Prasad, U. Shiva, et al. "Design and analysis of two throat wind tunnel." International Journal of Mechanical and Production Engineering Research and Development 7.4 (2017): 381388.

10. Jeffrey D. Flamm, Karen A. Deere, Bobby L. Berrier, Experimental study of a dual throat fluidicthrust vectoring nozzle concept, 41 st AIAA/ASME/SAE/ASEE joint propulsion conference \& exhibit, 10-13 July 2005, Tucson, Arizona. AIAA 20053503.

11. Balakrishna, B., S. U. S. H. M. A. Indana, and R. P. Reddy."Investigation of supersonic flow through conical nozzle with various angles of divergence." International Journal of Mechanical Engineering 2.1 (2013): 9-16.

12. B. L. Smith, A. Glezer, Jet vectoring synthetic jets, J.Fluid Mesh, Vol. 458, 2002:1-34. 


\section{AUTHORS DATA}

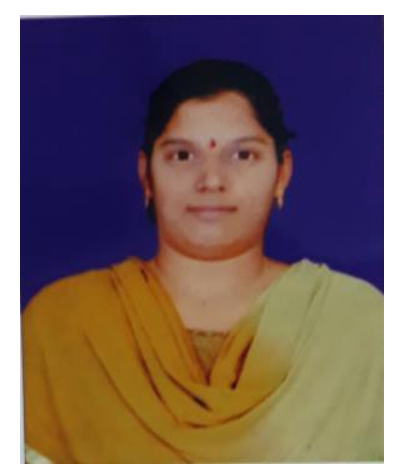

FIRST AUTHOR: Ms. Bapathu Bindu Mounica has completed her B. Tech (Mechanical engineering) from NIET affiliated by JNTUK and M. Tech (Aerospace engineering) from MRCET. Area of interest in Fluid Mechanic, CFD. 

